\title{
Augmented Reality Based Bee Drift Analysis: A User Study
}

\author{
Huyen Nguyen*, Sarah Ketchell ${ }^{\dagger}$, Ulrich Engelke*, Bruce H. Thomas* and Paulo de Souza* \\ *Data61, Commonwealth Scientific and Industrial Research Organisation (CSIRO), Sandy Bay TAS 7005, Australia, \\ emails: huyen.nguyen@csiro.au, ulrich.engelke@csiro.au, paulo.desouza@csiro.au \\ ${ }^{\dagger}$ School of Engineering \& ICT, University of Tasmania, Sandy Bay TAS 7005, Australia, email: sarahk8@utas.edu.au \\ ${ }^{\ddagger}$ School of Information Technology and Mathematical Sciences, University of South Australia, \\ Mawson Lakes SA 5095, Australia, email: bruce.thomas@unisa.edu.au
}

\begin{abstract}
We present HoloBee, a system for visual analytics of bee drift data using a head-mounted augmented reality interface (Microsoft HoloLens). HoloBee facilitates interactive manipulation and exploration of the bee activity data on 3D geo-spatial terrains to gain insights into the drifting behaviour of honey bees in their natural habitat. We conducted a user study to compare the task performance and user experience of HoloBee with a conventional desktop application. The results show that HoloBee allows for solving tasks in a similar time and accuracy compared to the desktop interface, despite participants' having significantly more experience interacting with a desktop interface. We received positive feedback from a subset of participants on the natural and intuitive interactions that HoloBee provides, while others preferred using the desktop application. We discuss the implications and limitations of these results and provide guidance for future work in this direction.
\end{abstract}

Index Terms-augmented reality, visual analytics, data visualisation, user study, bee drift

\section{INTRODUCTION}

With the advent of new immersive technologies and especially head-mounted displays, virtual reality (VR) and augmented reality (AR) are at the verge of becoming a commodity for a wide range of end users. While VR and AR headsets have been often considered in various applications (e.g. gaming, education, or manufacturing), only recently have they started to attract attention as viable interfaces for effective data exploration. Immersive analytics [1] of big, complex data sets may benefit from large screen real estate, natural interactions, and more engaging experiences, that are ultimately expected to result in improved reasoning and informed decision making. Augmented reality comes with the added bonus of facilitating analytics embedded in the real world context [2] and is expected to be highly beneficial for collaboration amongst several analysts to explore the same problem.

While all the arguments may speak for immersive technologies as visual analytics interfaces, empirical evidence is needed to really support these claims. In this paper, we aim to contribute to this cause by reporting on a user study that we performed for a state-of-the-art AR headset, the Microsoft HoloLens ${ }^{1}$. Through this study, we aim to provide some early pieces of evidence how a head-mounted AR display

${ }^{1}$ https://www.microsoft.com/microsoft-hololens/en-us

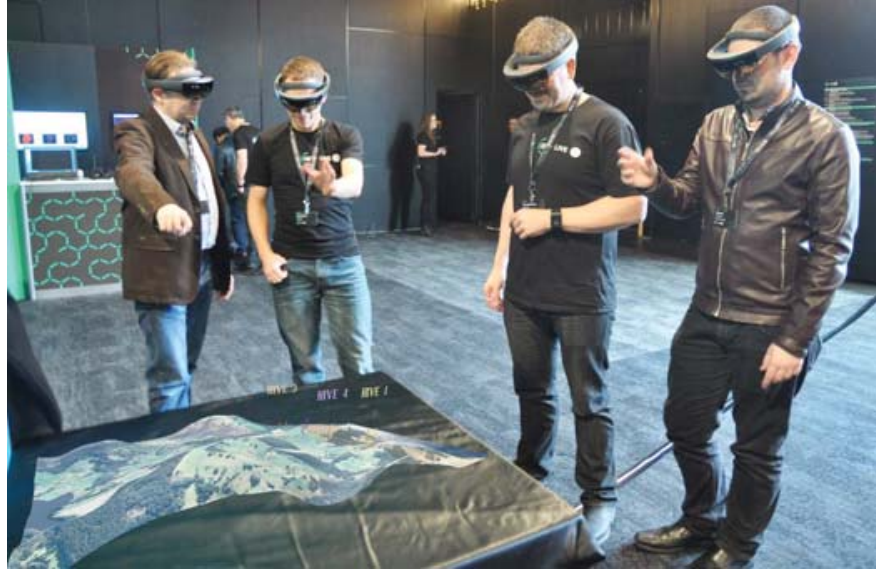

Fig. 1: Users explore the bee drift data using HoloBee.

performs in comparison to a conventional desktop interface for the exploration of scientific spatial data. We are considering a specific system called HoloBee that we implemented for interactive exploration of honey bee activity data derived from micro sensors attached to the bees. HoloBee is essentially a HoloLens-based AR interface sitting on top of a visual analytics framework that allows users to extract and analyse multiple activity measures from the sensor data [3]. HoloBee allows for end users to interactively explore bee activity data in a 3D spatial visualisation of their natural environment (see Fig. 1). Ultimately, it should facilitate collaboration of several analysts at co-located or remote locations [4].

In the study presented in this paper, we are focusing on one specific bee activity measure, bee drift, which relates to the behaviour of a bee leaving one colony to join another in a different hive [5]. Within this context, we aim to investigate task performance and user experience of HoloBee relative to a conventional desktop interface. We received positive feedback from a subset of participants on the natural and intuitive interactions that HoloBee provides, while others preferred using the desktop application. We discuss the implications and limitations of the results of our study and provide guidance for future work in this direction. 


\section{RELATED WORK}

As spatial data often needs to be investigated in three or more dimensions, conventional 2D monitors used for data visualisation and interaction may limit our experience and insights into the data. Advances in technology of many mobile display devices for AR can widely support data visualisation and visual analytics works [6]. Specifically for geo-spatial and temporal data, it has been demonstrated that AR can help users to acquire knowledge about spatial cognition phenomena [7]. Three types of spatial knowledge are mainly considered: procedural knowledge which is necessary for the navigation and wayfinding in geographic space, declarative knowledge which contains the information about geographic space and its elements, and configurational knowledge which relates to positions, orientations, distances, and relationship between elements in the space [8], [9]. AR-based application can put the $3 \mathrm{D}$ content in its natural settings and unburden users of desktop metaphors and the ambiguity between mouse clicks, button presses and their implications of interactions throughout the manipulation process.

Several studies have been conducted to identify through empirical work if AR interfaces provide advantages over 2D interfaces in the range of spatial data visualisation. Hedley [10] shows that the AR technology helped users to increase the performance of perceptual and task-based activities in geographic visualisation. For the Construct3D system by Kaufmann et al. [11], the usability of an AR application on a head-mounted display was rated higher than a desktop-based application, despite technical limitations such as software robustness, latency or cyber-sickness. MapLens - a mobile augmented reality system providing digital information overlaid on top of the view of physical maps - has been developed and compared to a standard 2D mobile map, which facilitates cooperative navigation and pathfinding [12]. Recently, Lee et al. [13] performed a study to compare the performance of using ARbased 3D hologram with 2D-based training in spatial direction and environmental cognitive capacity. It shows that the mental rotation ability improved through the use of the AR-based training system.

With recent advances in holographic display technology, there is a potential for greatly advancing the information visualisation and data analytics fields. The HoloLens offers a unique set of functionalities that allows users to easily and naturally manipulate data holograms through rotation, scale, reposition operations by gaze, gestures, voice commands or a remote control. With its own self-sufficient computer, the cordless capability of the HoloLens provides users the possibility of moving freely in space to observe scenes from different perspectives. The HoloLens is still relatively new and only limited prior research using this system exists. Chen et al. [14] describes a collaboration design between HoloLens and other devices such as tablets or PCs through Skype ${ }^{2}$. Mahfoud et al. [15] explore the benefits of using gaze-directed visualisation in mixed-reality using the HoloLens compared

\footnotetext{
${ }^{2}$ https://www.skype.com
}

to desktop applications. Voinea et al. [16] propose a system for HoloLens which enables users to effectively learn about biomechanics. The immersive and natural interaction is identified to be of great value, but mobility issues and limited access to the application when deployed solely to the HoloLens are identified as limitations. To the best of our knowledge, there are no studies to date that address specifically the performance and user experience of HoloLens in the context of scientific visualisation.

\section{HoloBeE SyStem}

In this section we briefly describe the design and implementation of the HoloBee system.

\section{A. Bee Drift Data}

We attach miniaturised RFID tags to the backs of honey bees to study their behaviour in the natural environment. We cannot track them in space during flight but only at readers that are installed at hives and feeder stations. Each bee has its unique ID. Bee activity recordings have been collected throughout a six month experiment in 2015 with 8,230 tagged honey bees in the Geeveston and Cairns Bay sites in Tasmania, Australia.

In this work we focus solely on one specific bee behaviour measure, bee drift, which represents the phenomenon of a bee moving from one hive into another hive. This measure could be used to predict bee health, for instance, in case of bees transmitting pathogens from one hive to another. It also reflects hive management issues because drifting often occurs when bee colonies are kept in close proximity to one another [5]. Hive drift is typically a rare event for which reason associated readings are very low as compared to overall bee activity.

\section{B. System Design and Implementation}

The HoloBee system has been specifically designed taking into account features and limitations of the Microsoft HoloLens. Many design aspects, however, are easily transferable to other AR headsets in the future by adapting the design constraints. A detailed description of HoloBee can be found in [17], but for the readers, convenience we provide a brief overview here.

The interactive visual interface of the HoloBee was built to facilitate an effective data exploration process for both novice and expert users. It allows users to easily map data into the geo-spatial context of 3D space and to move and interact naturally and intuitively within. The Overview+Detail paradigm [18] and Cognitive Task analysis [19] have been used as guidelines to build a UI that aids the user to easily situate the information of a particular experiment in a global context. Therefore, there are different levels of details of the data exploration: a global map that shows all experiment sites, and site scenes that visualise hive drift as well as visits to feeder stations, as shown in Fig. 2a and Fig. 2b, respectively. Although the bee visits to feeder stations are not classified as bee drift, they are still included in this visualisation because 


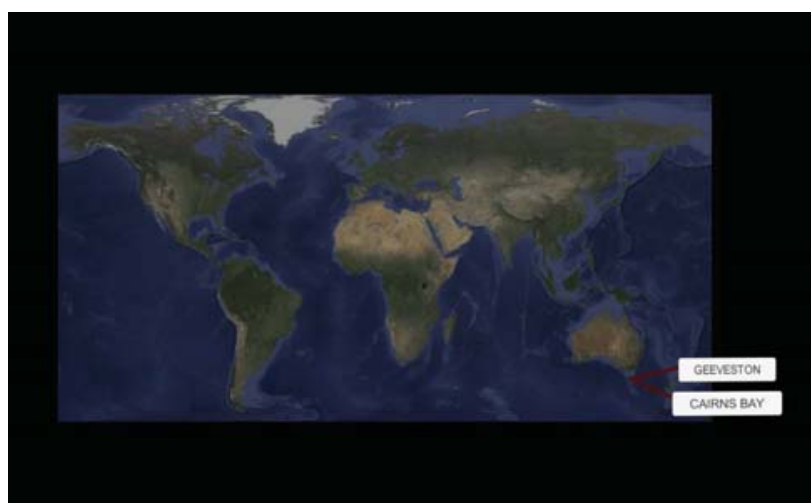

(a)

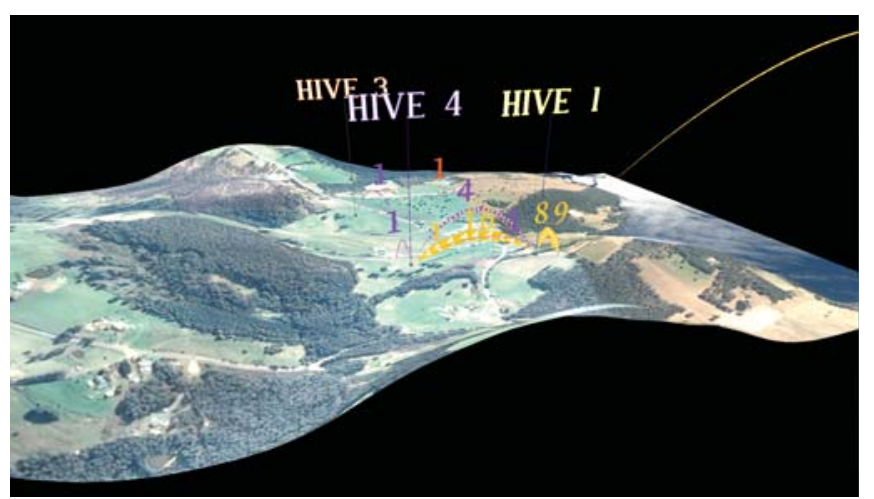

(b)

Fig. 2: Global scene (a) of the system with all experiment sites labelled and site scene (b) that visualises hive drift as well as visits to feeder stations in a 3D geo-spatial map.

they exhibit the same analytics and visualisation properties as drift between hives.

The Microsoft HoloLens provides a wide range of natural and intuitive interaction techniques that facilitate high task performance and user experience. We implemented voice control, gaze tracking, and gesture recognition as means for the user to interact with the system. Using the voice control, the user can select months for which data should be visualised and can switch between scenes. Gesture recognition engine of the HoloLens is still limited to only two gestures, one to make selections on interactive elements of the scenes and the other to return to the main menu. The gaze tracking interaction is complementary to the gesture recognition engine, which indicates on which interactive elements the user wants to initiate actions.

Besides the aforementioned interaction techniques, HoloBee also assists the user in exploring the data by integrating visual and auditory feedback mechanisms into the system. The visual feedback including directional pointers and a visual cursor that is dynamically mapped onto the $3 \mathrm{D}$ world make the user easily focus on the main part of the data visualisation as well as be aware of the limited field of view of the HoloLens. The auditory channel, on the other hand, provides supplementary information to the user during data exploration without the need for the user to directly interact with system controls. This is an effective way of providing detailed information of the current site such as name, position on the global map, number of hives, current timeline, etc. Moreover, a "Help" menu is always visible to be accessed during runtime and its content can be context-driven.

Bee drift visualisation was created by arcs drawn between two hives or between one hive and a feeder station (see Fig. 2b). The bee drift representations were encoded with color, size, arrow symbols, and numbers to show different properties: a source hive, a destination hive, the number of bees drifting.

The HoloBee system was developed for Microsoft HoloLens using Unity and Visual C\#. It connects to the bee database via
SQLite plugins. Moreover, line renderer component in Unity was used to model drift between hives. The Geeveston and Cairns Bay terrains were extracted and textured from Google Earth.

\section{USER STUDY}

We conducted a user study to evaluate task performance and user experience of HoloBee for exploration of bee drift. For benchmarking purposes, we compare HoloBee to a conventional desktop interface implementation. This section discusses in detail the study design and results.

\section{A. Study Design}

1) Task and Conditions: A set of three questions was asked for each interface, which are summarised in Table I. The questions were chosen such that the task complexity increases from Q1 through Q2 to Q3. Specifically, Q1 requests only a binary answer (Yes/No) based on bee drift analysis in one month. Question Q2 requests a choice from six possible answers, based on bee drift analysis on all six months of the experiment. Finally, question Q3 requests six answers, based on bee drift analysis on all six months of the experiment. Questions within each category are considered the same in task complexity. Given the aforementioned, we have two independent variables in the user study, Interface type $I$ and Question type $Q$ (see Table II).

2) Hypotheses: Provided that the AR interface offers a more natural means for the user to interact with the system via voice control and gesture recognition, we define two hypotheses as follows:

H1 It takes less time and effort for the user to solve analytics tasks with the HoloBee interface than with the Desktop interface.

H2 The user experience is better for the HoloBee interface than for the Desktop interface in terms of intuitiveness, ease of use and naturalness. 
TABLE I: Overview of the tasks that the participants needed to solve and the respective possible responses for each. The task complexity increases from Q1 through Q2 to Q3.

\begin{tabular}{lll}
\hline ID & Question & Possible responses \\
\hline \hline Q1 & $\begin{array}{l}\text { Is there drift from Hive 1 to any other Hive in April? } \\
\text { Is there drift from Hive 4 to any other Hive in March? }\end{array}$ & Yes / No \\
\hline Q2 & $\begin{array}{l}\text { Which month has the least amount of drift in the Cairns Bay site? } \\
\text { Which month has the most amount of drift in the Geeveston site? }\end{array}$ & January / February / March / April / May / June \\
\hline Q3 & $\begin{array}{l}\text { How many bees moved from Hive 4 in each month of the whole period? } \\
\text { How many bees moved from Hive 3 in each month of the whole period? }\end{array}$ & Six integer values, one for each month \\
\hline
\end{tabular}

TABLE II: Independent variables for the user study.

\begin{tabular}{ll}
\hline Independent variables & Values \\
\hline Interface type $I$ & Augmented Reality (AR) / Desktop \\
Question type $Q$ & $\mathrm{Q} 1 / \mathrm{Q} 2 / \mathrm{Q} 3$ \\
\hline
\end{tabular}

3) Experiment Environment and Technical Setup: The experiment was set up in a large meeting room with sufficient space for the participants to walk around a central table when using the AR interface. We intentionally chose a meeting room as an environment in which the AR interface may be used in a realistic context, wherein several users can jointly participate in solving analytics tasks. The desktop interface was setup in a corner of the same room for the participants convenience to move between experiment conditions. The light in the room was kept dim throughout the experiment.

For the AR condition, we used the HoloBee system described in Section III with the 2016 Microsoft HoloLens Development Edition. Video was recorded through the HoloLens for post-hoc analysis of the user interactions. The screenshot of the AR interface can be seen in Fig. 3.

The Desktop interface consists of a Microsoft Windows 10 workstation with a monitor, a keyboard, and a mouse. The same 3D visualisation as presented in the HoloLens was presented on the 2D screen instead. Several necessary alterations were made to the application to account for different interaction techniques (using keyboard and mouse instead of voice, free hands, and gaze). The alterations made include adding key presses for controls, as well as mouse clicks and scrolling in place of moving physically. The application also records time, key presses, and mouse clicks for evaluation purposes. Table III provides details of the actions initiated through the command language of the AR and Desktop interfaces.

4) Stimuli: We used the microsensing data collected from an experiment that took place over six months from 12th January 2015 to 25th June 2015 in Tasmania, Australia. Four hives were placed at two sites in this experiment, one in Geeveston (hive 2) and three in Cairns Bay (hives 1, 3, and 4). There were 8,230 bees that have been tagged over the six month period. After the post-processing step of the data, we have recorded in total 4,117,923 bee detections from four hives. The number of bee drift readings of each month derived from the bee detection data is shown in Table IV.

5) Participants: Fourteen participants (five females) aged from 21 to 73 (mean: 38.14, std: 14.24) took part in this experiment. They were recruited amongst CSIRO staff and

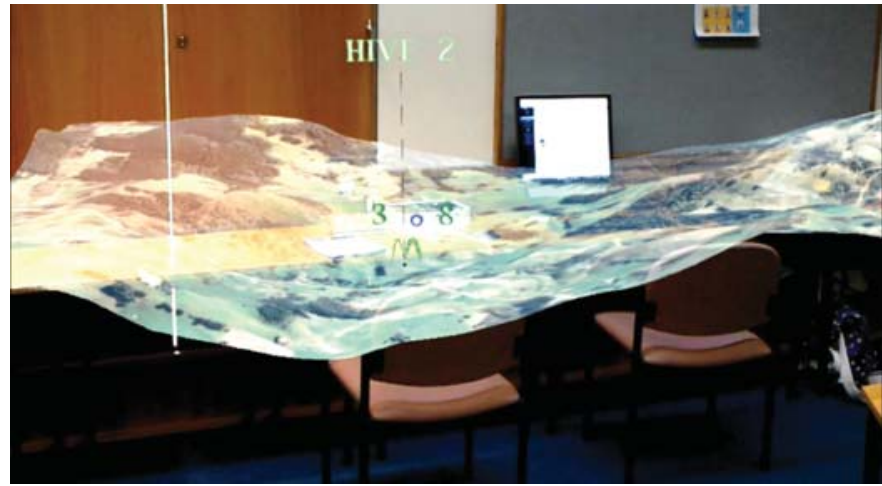

Fig. 3: Drift data of Geeveston site on the AR interface. On the Desktop interface, the live background is replaced by black colour.

visiting students from Australian universities. Six of them had limited experience with HoloLens and holograms before participating in our study. Four were students in computer science and information technology and six were research scientists and engineers at CSIRO. The remainder of the participants worked in education or administration related jobs. We did not recruit any bee keepers or entomologists for our study because the tasks were simple enough to be solved by non-experts. All participants were volunteering their time and received no reimbursement beyond light refreshments.

6) Experiment Procedure: Each experiment session consisted of an initial instruction followed by two evaluation phases (AR and Desktop interface). Each evaluation phase consisted of training, task solving, and user feedback.

At the beginning of the session, the purpose of the experiment was explained to the participants and any questions were answered. Participants were informed that Human Research Ethics approval had been obtained for the study and that any concerns can be addressed to the respective committee. Participants were then asked to carefully read and sign a consent form.

Following the instruction phase, participants then started the first of two evaluation phases. The order of AR or Desktop interface was counter-balanced. The training session for each interface explained the respective functionality while participants were exploring a specific training data set. Participants were encouraged to explore the 3D world and look at the data from different viewpoints to familiarise themselves with the manipulation and interaction methods of the interfaces. 
TABLE III: Command language for the AR and Desktop interfaces and the respective actions they initiate.

\begin{tabular}{|c|c|c|c|c|c|c|}
\hline \multirow[t]{2}{*}{ Scene } & \multicolumn{3}{|c|}{ Augmented Reality } & \multicolumn{2}{|c|}{ Desktop } & \multirow[t]{2}{*}{ Action } \\
\hline & Voice & Gesture & Moving & Mouse & Keyboard keys & \\
\hline \multirow[t]{2}{*}{ Global } & "Cairns Bay" & Select "Cairns Bay" & & Left click "Cairns Bay" & & Enter Cairns Bay site \\
\hline & "Geeveston" & Select "Geeveston" & & Left click "Geeveston" & & Enter Geeveston site \\
\hline \multirow[t]{2}{*}{ Site } & "Main menu" & Select "Menu" button & & & "m" & Return to Global scene \\
\hline & "Display" + Month & & & & "1", "2", ,.., "6" & Display monthly data \\
\hline \multirow[t]{4}{*}{ Any } & & & Forward/backward & Scroll wheel & & Zoom in / out \\
\hline & & & Around the scene & Click right and drag & & Rotate the scene \\
\hline & & & Left/right & Click left and drag & & Translate the scene \\
\hline & "Finish" & & & & "f" & Complete task \\
\hline
\end{tabular}

TABLE IV: Number of bee drift detections over the six month period of the four hives at Geeveston and Cairns Bay sites.

\begin{tabular}{l|c|c}
\hline Month & From - To & Drift detections \\
\hline \hline January & Hive 1 - Hive 3 & 1 \\
& Hive 2 - Hive 1 & 1 \\
& Hive 4 - Hive 3 & 1 \\
\hline February & Hive 1 - Hive 4 & 13 \\
& Hive 2 - Hive 1 & 4 \\
& Hive 4 - Hive 1 & 14 \\
\hline March & Hive 1 - Hive 2 & 3 \\
& Hive 1 - Hive 4 & 16 \\
& Hive 3 - Hive 1 & 1 \\
& Hive 4 - Hive 1 & 4 \\
& Hive 4 - Hive 3 & 1 \\
\hline April & Hive 1 - Hive 2 & 1 \\
& Hive 1 - Hive 3 & 2 \\
& Hive 3 - Hive 1 & 1 \\
\hline May & None & 0 \\
\hline June & None & 0 \\
\hline
\end{tabular}

During the main evaluation, participants were asked to answer the questions as presented in Section IV-A1. Participants were required to find the answers through the manipulation and exploration of the visualised data. Results were verbally reported to the experimenter who recorded them in a spreadsheet. At the end of the two evaluation sessions, the participants were asked for feedback on their overall user experience using the two interfaces by filling out a questionnaire. Each experiment session duration was approximately 30 minutes.

7) Performance and User Experience Metrics: For the evaluation of the two interfaces, we measured task performance through quantitative metrics and overall user experience through a questionnaire.

Three task performance metrics were recorded: completion time, number of interactions, and correctness of answers. Completion time is a proxy for efficiency of using any of the two interfaces for solving a task. It was recorded from the moment the participants initiated a task until they finished a task. Since the two interfaces are completely different in interaction paradigm, the number of interactions that the participants have invoked to manipulate the visualised data were recorded as a proxy for effort of solving a task. For the AR interface, these interactions included voice commands and hand gestures and were established post-hoc through video analysis. For the Desktop interface, interactions included mouse scroll, mouse click, and key press. These interactions were directly recorded by the system. Lastly, correctness of answers was evaluated in post-hoc analysis by comparing given answers to the correct answers.

After the evaluation sessions, participants were asked to rate their user experience on a 7-point Likert scale according to the following criteria: intuitiveness, ease of use, comfort, naturalness, efficiency, and overall preference for a particular interface. Demographic information was also recorded detailing the age, gender, and the experience of the participants in using HoloLens and hologram visualisations.

\section{B. Results}

1) Completion Time: We have two independent variables, Interface type $I$ and Question type $Q$, which could influence the completion time of two consecutive phases of a data analytics trial. Due to the voice recognition problem of HoloLens when the participants used vocal controls to explore the data, the time that they had to spend to repeat these commands until they were accepted was discarded from the final completion time. A repeated-measure two-way ANOVA for balanced design and within-subject factor was used to evaluate the interaction of the two factors on the completion time.

The result revealed that there was no significant interaction effect between the factors $I$ and $Q$ on the completion time $[F(1.41,18.36)=0.88, p=0.43]$. Since the Mauchly's test of sphericity was statistically significant $[p<0.05]$, the Greenhouse-Geisser correction was used to adjust the degrees of freedom of F-ratio. The mean completion time with its standard deviation is shown in Fig. 4 for both interfaces and all three question types.

The result of the test for the main effect of the Question factor showed a significantly independent effect on the completion time $[F(1.33,17.27)=12.24, p<0.05]$. In other words, the significant main effect of the Question factor on the completion time showed that our study design using three types of questions correctly reflected the different levels of task complexity, which in turn required different lengths of time to solve. However, no significant main effect of the Interface factor on the completion time has been found $[F(1,13)=0.01$, $p=0.92]$. The results showed that there was no difference in the completion time between the AR and Desktop interface.

2) Correctness of Answers: The answers by the participants were evaluated manually and correct answers were scored 1 


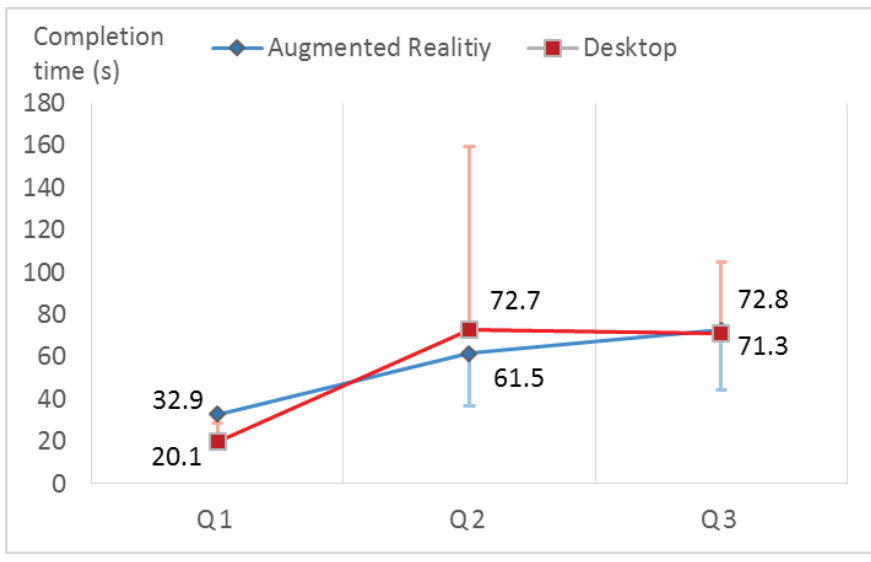

Fig. 4: Mean completion time for both interfaces and all three question types.

while incorrect answers were scored 0 . The resulting data was analysed using repeated-measures two-way ANOVA. Significant interaction $[F(2,26)=4.58, p=0.01]$ of the two factors $I$ and $Q$ suggests that the main effects of the two factors should be ignored and the simple main effects for the two independent variables were investigated. Three subsets of our dataset which correspond to the three sets of questions (Q1, Q2, Q3) were evaluated to investigate the impact of the Interface factor $I$. An ANOVA analysis has been run for each subset with the Interface factor being the independent variable. Therefore, the Type I error for the p-value is calculated by dividing the typical 0.05 alpha level by three which is equal to 0.017 . At a new alpha level of 0.017 , the result revealed that there was insufficient statistical support for an interface difference in each set of questions. Fig. 5 represents the interaction plot of the answer score over all given answers for three sets of questions using the two interfaces.

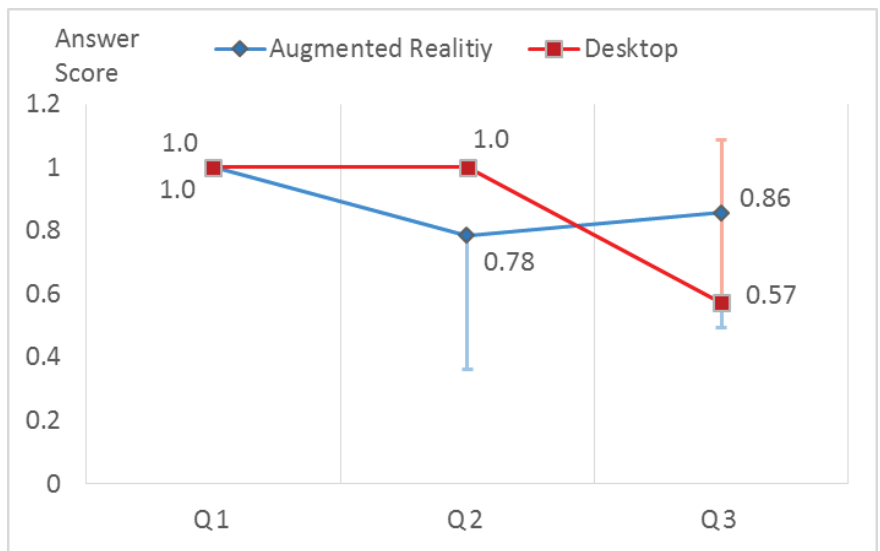

Fig. 5: Correct answers ratio for both interfaces and all three question types.

3) Interaction Record: The repeated-measure two-way ANOVA for balanced design and within-subject factor was also used to analyse the interaction of the two factors $I$ and $Q$ on the Interaction variable. We did not find any significant interaction between these two factors $[F(2,26)=2.06, p=$ 0.13]. The Mauchly's Test of Sphericity was not statistically significant $[p=0.15]$. Therefore, the degrees of freedom of $F$-ratio remained intact. Fig. 6 shows the interaction plot of these two factors on the Interaction variable.

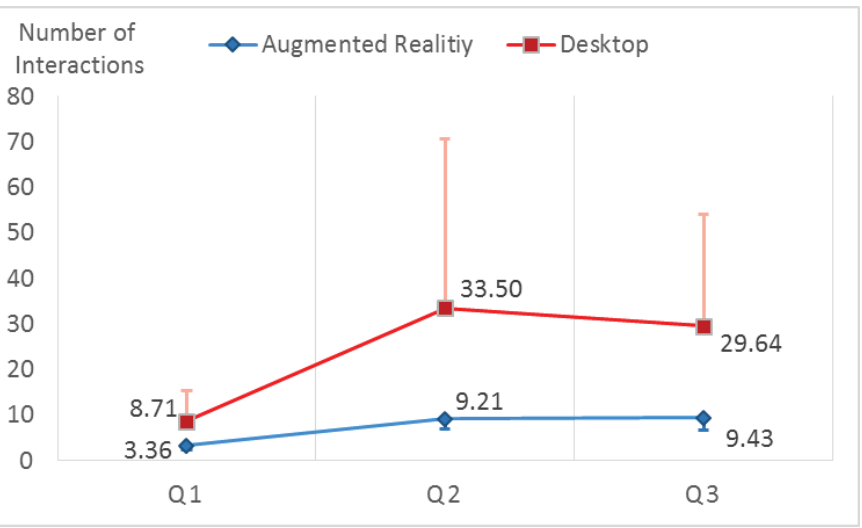

Fig. 6: Average number of interactions for both interfaces and all three question types.

The test for the main effects of the two factors $I$ and $Q$ has been done to investigate their separate effect on the Interaction variable. Each factor had a significantly independent effect: $[F(2,26)=5.81, p<0.05]$ for the Question factor and $[F(1$, $13)=17.17, p<0.05]$ for the Interface factor. From these results and the interaction plot, we can conclude that using the $\mathrm{AR}$ interface required significantly fewer operations to interact with the data than the Desktop interface.

4) Subjective Questionnaire: Subjective questionnaire answers were gathered and the data was analysed using a Wilcoxon signed-rank test with continuity correction. Results of the questionnaires were crossed for comparison.

We did not find any significant difference between the $\mathrm{AR}$ and Desktop interfaces on the attributes intuitiveness, ease of use, comfort, naturalness and efficiency. The detailed percentage distribution of the evaluation of the two interfaces for each criterion can be found in Fig. 7. The mean values and the p-values from the statistical analysis are reported in Fig 8.

An overall rating between the two interfaces on the 7-point Likert scale was also analysed and its result is illustrated in Fig. 9. We can observe that the entire spectrum of preference is covered, with some participants preferring the AR interfaces while others prefer the Desktop interface.

\section{Discussion}

HoloBee is an AR-based visual analytics system for the exploration of bee drift in a natural context. A user study was performed to formally evaluate task performance and user experience using HoloBee. Statistical analysis showed that HoloBee could not outperform its desktop version by means of completion time and correctness of answers. However, HoloBee required significantly fewer interactions by the user to solve the given tasks. This could be explained by the fact 
Intuitiveness

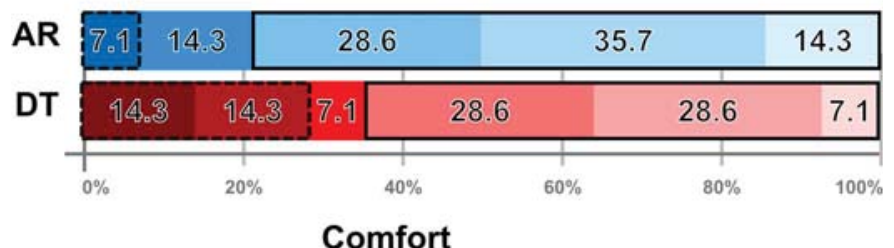

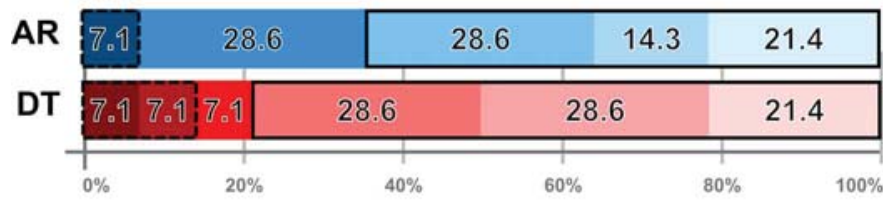

Efficiency
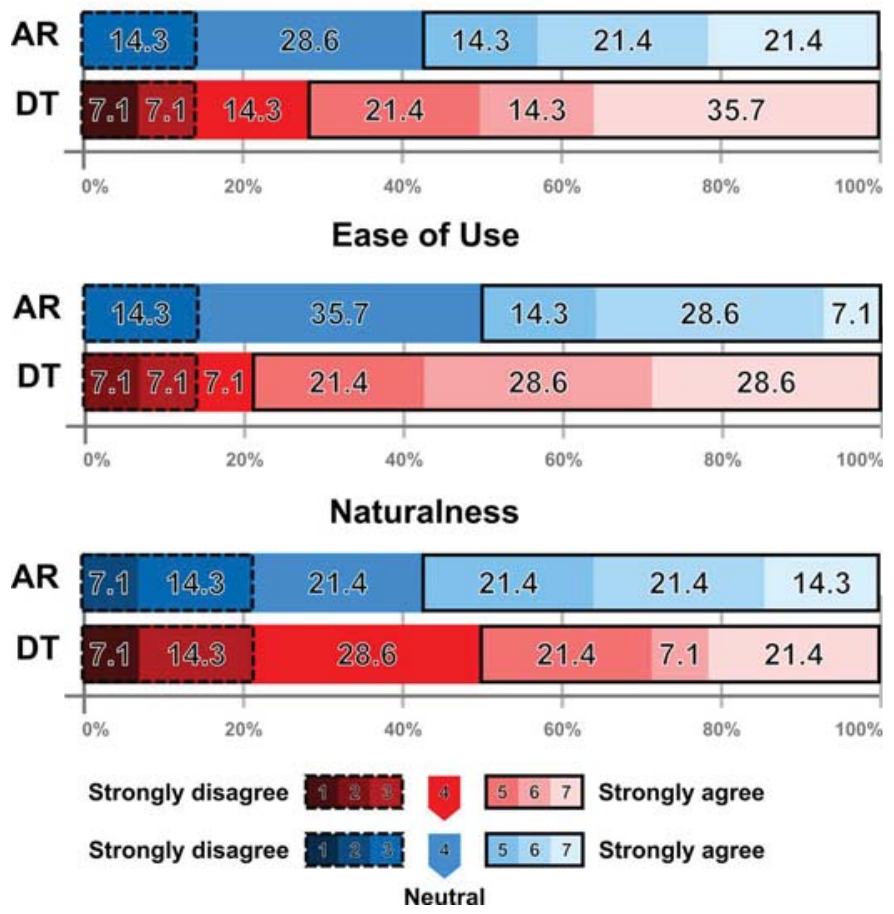

Fig. 7: Questionnaire results for both interfaces (Augmented Reality and Desktop) showing the percentage distribution of all attribute ratings on a 7-point Likert scale.

that through simple voice commands, selection gestures, and the ability of moving around the scene, the user can easily manipulate the visualised data in a natural and intuitive approach. Completion time, however, has not been significantly affected. We can speculate that this is attributed to the time taken to move in 3D space when using the HoloLens, which we did not quantify as an interaction. Due to this limitation, we are therefore hesitant in accepting or rejecting hypothesis H1.

We cannot draw any definitive conclusions based on the statistical analysis of the questionnaire and can therefore not accept hypothesis $\mathbf{H 2}$ that HoloBee improves overall user experience on a set of given attributes. The questionnaire responses indicate that a subset of participants preferred the

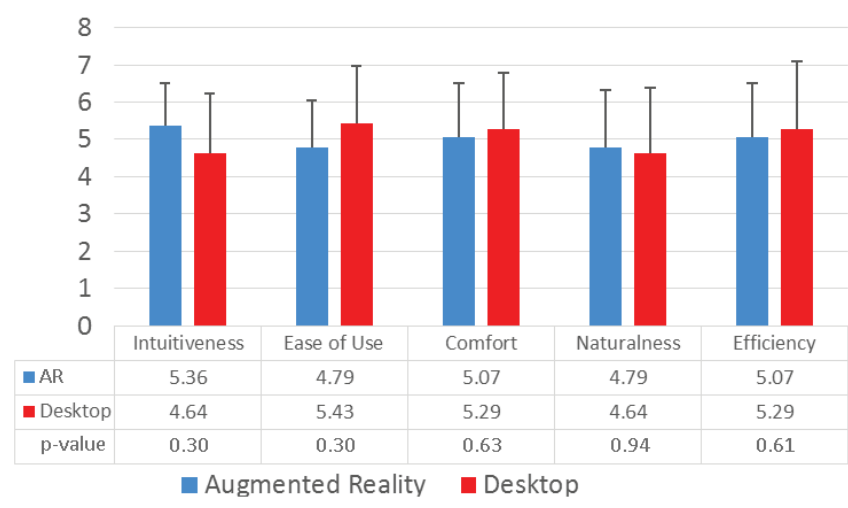

Fig. 8: Mean scores and p-values of the subjective data from the questionnaire.

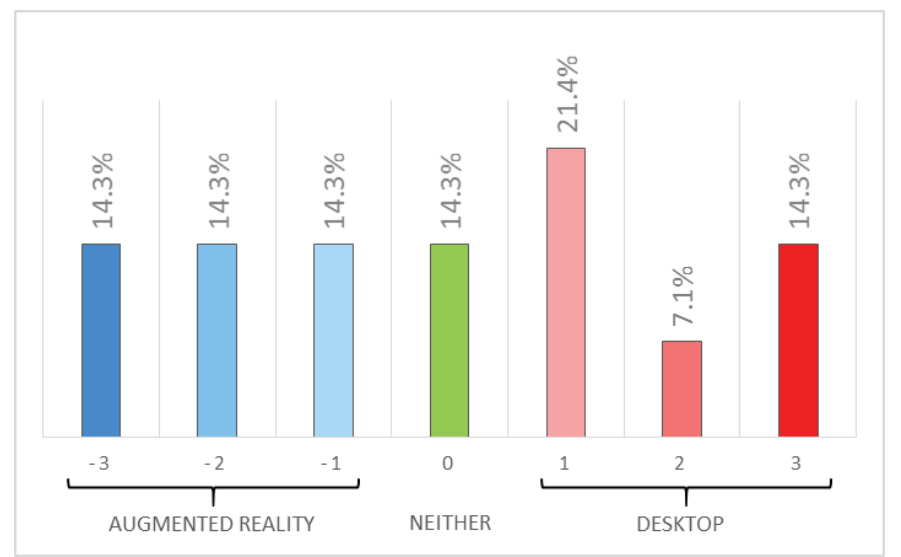

Fig. 9: Percentage distribution of subjective preference between the Augmented Reality interface and the Desktop interface (from -3 for the $\mathrm{AR}$ interface to 3 for the Desktop interface). Mean $=-0.07$ and $\mathrm{STD}=2.02$.

AR interface while others preferred the Desktop interface. We could not attribute this selection to any of the demographic data collected as part of the study (previous experience, gender, age). From short interviews with the participants of the study we still received positive feedback on the immersive experience using HoloBee. Most of the participants found that the training process was simple by only having to memorise four main types of voice commands with natural linguistic meanings behind them. The capability of freely walking around the scene to observe the data was also highly appreciated. According to several participants, looking at the bee drift data through a HoloLens device was found to be more exciting and engaging than through the conventional $2 \mathrm{D}$ monitor.

While we believe for the outcomes to be insightful and valuable for the performance of AR-based versus desktopbased analysis of bee drift (and spatial data more generally), we acknowledge several limitations of the study. Firstly, the scenarios and related tasks were not overly complex given the number of sites and hives we had available. More complex 
tasks might show more significant effects on the performance metrics as well as accentuate the experience that the participants have when using HoloBee. Secondly, we have focused on one very specific aspect of bee behaviour, bee drift. Ultimately, we intend for the system to also visualise other bee activity data, such as hive statistics and flight trajectories. Extending the complexity of the visual analytics pipeline may lead to deeper insights on the comparable usability of the ARand desktop-based interfaces. Thirdly, instead of interaction records, alternative complex measurements such as psychological approaches to evaluate users' effort of solving task may be used in the user study. Finally, given the disparate ratings on the user experience attributes, we need to involve a larger and broader user base to better understand the relationship of demographic background with interface preference.

Due to several limited capabilities of the HoloLens and most significantly, its narrow field of view, our user interface has been specifically designed to overcome these limitations, which takes into account the size, colour of visual elements in the scenes, vocal explanatory messages, simple but effective controls, voice recognition, etc. In the context of our study, the limited field of view may have had an impact on the performance metrics and likely the completion time as people would have to move around more to observe the scene compared to when a broader field of view would be available. With advancements in head-mounted AR technology, such limitation will likely disappear in coming years.

\section{CONCLUSION}

In this paper, we present HoloBee, a visual analytics system that allows users to analyse bee drift data through a headmounted augmented reality interface (Microsoft HoloLens). We conducted a formal user study to evaluate task performance and user experience when using HoloBee as compared to a conventional desktop interface. While completion time and accuracy of task solving remained on par between the interfaces, we were able to show that HoloBee requires significantly less interactions than the desktop interface. Opinions on user experience as measured through a questionnaire was divided between the participants of the study, with some preferring HoloBee and others preferring the desktop interface.

HoloBee can be easily expanded to include new types of data, analytics processes, and visualisation techniques. Moreover, collaborative analysis and in-situ analysis in outdoors environments are considered two important avenues to be further investigated. We will also include data from additional experiments as they become available and amend the visual analytics pipeline to allow for exploration of other bee activity measures, such as hive statistics and flight trajectories, as well as weather conditions.

\section{REFERENCES}

[1] T. Chandler, M. Cordeil, T. Czauderna, T. Dwyer, J. Glowacki, C. Goncu, M. Klapperstueck, K. Klein, K. Marriott, F. Schreiber et al., "Immersive analytics," in Proceedings of the Big Data Visual Analytics (BDVA), 2015. IEEE, 2015, pp. 1-8.
[2] N. ElSayed, B. Thomas, K. Marriott, J. Piantadosi, and R. Smith, "Situated analytics," in Proceedings of the Big Data Visual Analytics (BDVA), 2015. IEEE, 2015, pp. 1-8.

[3] U. Engelke, P. Marendy, F. Susanto, R. Williams, S. Mahbub, H. Nguyen, and P. de Souza, "A visual analytics framework to study honey bee behaviour," in Proceedings of the 2nd IEEE Data Science and Systems Conference (DSS). IEEE, 2016, pp. 1504-1511.

[4] H. Nguyen, P. Marendy, and U. Engelke, "Collaborative framework design for immersive analytics," in Proceedings of the 2nd International Symposium on Big Data Visual Analytics (BDVA). IEEE, 2016, pp. 1-8.

[5] J. B. Free, "The drifting of honey-bees," The Journal of Agricultural Science, vol. 51, pp. 294-306, december 1958.

[6] H. Slay, M. Phillips, R. Vernik, and B. Thomas, "Interaction modes for augmented reality visualization," in Proceedings of the 2001 Asia-Pacific Symposium on Information Visualisation (APVis). Australian Computer Society, Inc., 2001, pp. 71-75.

[7] B. E. Shelton and N. R. Hedley, "Exploring a cognitive basis for learning spatial relationships with augmented reality," Technology, Instruction, Cognition and Learning, vol. 1, no. 4, pp. 323-357, 2004.

[8] R. G. Golledge, "Cognition of physical and built environments," Environment, cognition, and action: An integrated approach, pp. 35-62, 1991.

[9] D. M. Mark and S. M. Freundschuh, Spatial Concepts and Cognitive Models for Geographic Information Use. Springer Netherlands, 1995, pp. 21-28.

[10] N. R. Hedley, "Empirical evidence for advanced geographic visualization interface use," in Proceedings of the 21st International Cartographic Conference (ICC), 2003, pp. 383-392.

[11] H. Kaufmann and A. Dünser, "Summary of usability evaluations of an educational augmented reality application," in Proceedings of the 2nd International Conference on Virtual Reality (ICVR). Springer Berlin Heidelberg, 2007, pp. 660-669.

[12] A. Morrison, A. Oulasvirta, P. Peltonen, S. Lemmela, G. Jacucci, G. Reitmayr, J. Näsänen, and A. Juustila, "Like bees around the hive: A comparative study of a mobile augmented reality map," in Proceedings of the SIGCHI Conference on Human Factors in Computing Systems (CHI). ACM, 2009, pp. 1889-1898.

[13] I.-J. Lee, C.-H. Chen, and K.-P. Chang, "Augmented reality technology combined with three-dimensional holography to train the mental rotation ability of older adults," Computers in Human Behavior, vol. 65, pp. 488 500,2016

[14] H. Chen, A. S. Lee, M. Swift, and J. C. Tang, "3d collaboration method over hololens ${ }^{\mathrm{TM}}$ and skype ${ }^{\mathrm{TM}} \mathrm{end}$ points," in Proceedings of the $3 \mathrm{rd}$ International Workshop on Immersive Media Experiences (ImmersiveME). ACM, 2015, pp. 27-30.

[15] E. Mahfoud and A. Lu, "Gaze-directed immersive visualization of scientific ensembles," in Proceedings of the Companion on Interactive Surfaces and Spaces (ISS Campanion). ACM, 2016, pp. 77-82.

[16] A. Voinea, A. Moldoveanu, and F. Moldoveanu, "Bringing the augmented reality benefits to biomechanics study," in Proceedings of the Workshop on Multimodal Virtual and Augmented Reality (MVAR). ACM, 2016, pp. 9:1-9:6.

[17] H. Nguyen, S. Ketchell, U. Engelke, B. Thomas, and P. de Souza, "Holobee: Augmented reality based bee drift analysis," in To appear in Adjunct Proceedings of the 16th IEEE International Symposium on Mixed and Augmented Reality (ISMAR). IEEE, 2017, pp. 1-6.

[18] A. Cockburn, A. Karlson, and B. B. Bederson, "A review of overview+detail, zooming, and focus+context interfaces," ACM Computing Surveys, vol. 41, no. 2, pp. 2:1 - 2:31, january 2009.

[19] B. Crandall, G. Klein, and R. R. Hoffman, Working Minds: A Practitioner's Guide to Cognitive Task Analysis, 1st ed. MIT Press, 2006. 